recibido: $02.12 .11 /$ aceptado: 12.03 .12

\title{
El efecto agenda sobre los temas de campaña en las elecciones generales de 2008
}

\author{
Maria Luisa Humanes \\ Universidad Rey Juan Carlos \\ María de los Ángeles Moreno \\ Universidad Rey Juan Carlos
}

\section{Palabras clave}

Agenda-setting, temas de campaña, consumo de medios, España

\section{Resumen}

Durante las campañas electorales el efecto agenda setting presenta un interés especial puesto que la información sobre los candidatos tiene un canal privilegiado en los medios. Estudiamos la transferencia de importancia sobre los temas de campaña en las elecciones de 2008 en España. Con los datos de la encuesta postelectoral del CIS se han obtenido modelos multivariables de la regresión logística para explicar las variables que contribuyen a dicho efecto. Los resultados confirman que el consumo de información no es el único factor en la atribución de importancia, sino que actúan junto a otros condicionantes relacionados con la experiencia vital del individuo. 


\title{
The agenda-setting effect and the campign issues in 2008 general elections
}

\section{Keywords}

Agenda-setting, campaign issues, media consumption, Spain

\begin{abstract}
During election campaigns the agenda setting effect is of particular interest because the media are a privileged channel for the information about the candidates. We studied the transfer of importance on the campaign issues in the 2008 elections in Spain. We used a logistic regression with the post-election survey data from CIS to explain the agenda setting effect. The results confirm that the information consumption is not the only factor by giving salience to issues, but act together with other constraints related to the individual's life experience.
\end{abstract}

\section{Autoras}

María Luisa Humanes [marialuisa.humanes@urjc.es] es doctora en Ciencias de la Información y profesora Titular de Universidad en el área de conocimiento de Comunicación Audiovisual y Publicidad en la Universidad Rey Juan Carlos (2002). Es autora de Algo más que periodistas y Teoría e investigación en comunicación social.

María de los Ángeles Moreno [mariaangeles.moreno@urjc.es] es doctora en Ciencias de la Información y profesora Titular de Universidad en la Universidad Rey Juan Carlos (2009). Es directora del título propio de Experto en Gestión y Evaluación de la Comunicación y del título propio de Experto en Planificación, Gestión y Comunicación de Eventos Empresariales. Forma parte del equipo de investigación del European Communication Monitor. 


\section{Introducción}

La mayor parte de los estudios sobre agenda setting se han diseñado sobre la hipótesis de la correlación entre los temas destacados por los medios y los temas mencionados por los ciudadanos como los más importantes o los más preocupantes (en el caso de la agenda personal y/o pública) o por los actores políticos (agenda política). La abundante evidencia empírica en diversos contextos corrobora la hipótesis de un efecto específico del contenido. En un paso más, se ha sumado a esta explicación la asociación con los datos del mundo real para comprobar la asociación entre el desarrollo de los hechos sociales y su reflejo en los medios. Esta perspectiva se acompaña de una definición de la agenda setting como un efecto de nivel agregado al considerar la suma de las respuestas de los individuos, de tal forma que el contenido de los medios determinaría la agenda de la comunidad y no la de cada individuo (Kosicki, 1993: 103; Zhu y Blood, 1996: 101). Wanta (1997) presenta junto al modelo de transferencia del mensaje, que sitúa a los medios en el centro de la influencia, el modelo de la activación del conocimiento, que matiza el poder de los medios introduciendo más variables explicativas (1997: 17-19). Aunque en su obra los factores que se suman como determinantes del establecimiento de agenda se consideran de manera separada, y no en un modelo multivariable, nos parece un punto de partida valioso. La introducción en los análisis de más variables independientes para explicar el peso real de los medios en el establecimiento de las agendas, sean éstas públicas o políticas, garantiza no caer en la idea un modelo general de carácter lineal y de efectos fuertes (Walgrave, Soroka y Nuytemans, 2008: 815; Zhu et al. 1993).

El efecto agenda setting se ha desarrollado al menos en tres direcciones: investigando las relaciones de causalidad, los determinantes del efecto y el contexto sustantivo (Zhu y Blood, 1996: 109). El principal escollo para resolver el problema de la causalidad se encontraba en el nivel agregado de la unidad de análisis en las encuestas, de modo que resulta imposible comprobar el efecto agenda en cada individuo. Iyengar y Kinder (1987), con un diseño experimental, así como Protess et al. (1985), han testado en el laboratorio el modelo de transferencia de importancia desde el contenido mediático a la opinión de la gente. Una alternativa a los estudios de laboratorio ha sido la utilización de modelos de ecuaciones estructurales - técnica multivariable que permite descubrir relaciones de causalidad lineales - y el análisis de series temporales. Sobre este último supuesto Kosicki afirmaba que, aunque muchos autores definían la agenda setting como un proceso dinámico, la mayoría de los estudios se basaban en datos recogidos en un momento concreto (1993: 106).

La segunda de las tendencias, también denominada contingent theory, sostiene que las características de la audiencia, de los temas y de los medios de comunicación intervienen en el efecto agenda. Respecto al público, la necesidad de orientación, el interés por la campaña, la alineación política, la educación u otras características sociodemográficas deben considerarse como variables que 
sirven para descartar efectos espurios. Este tipo de diseños se enmarcan en el nivel micro (Kosicki, 1993: 106).

Las características de los temas como determinante en el efecto agenda fue puesto de manifiesto en primer lugar por Zucker (citado en Walgrave y van Aelst, 2006: 93), y plantea que la influencia de los medios varía en función de los llamados obtrusive and unobtrusive issues. Aquellos asuntos de los cuales se tiene experiencia directa (obtrusive) son menos sensibles al efecto agenda, mientras que la influencia de los medios se incrementa cuando se trata de asuntos cuya fuente de conocimiento principal son los medios. El paro, la vivienda, los problemas económicos o la inmigración serán asuntos menos sensibles a la influencia de los medios que la política exterior, el terrorismo o las guerras, como ha puesto de manifiesto la literatura existente (McCombs, 2004: 61-62).

Se retoma la consideración de la experiencia y las relaciones personales como variables moderadoras del efecto agenda (McCombs, 2004: 60). Más concretamente se incide en el concepto de necesidad de orientación, en el que se combinan la experiencia personal, el uso de los medios y las conversaciones sobre el tema, puesto que el nivel de tal necesidad determina en qué grado la comunicación interpersonal refuerza o contraviene la influencia de la agenda mediática (McCombs, 2004: 65). Sólo rescataremos el estudio de Demers (1993), quien afirmaba que en los primeros estudios de agenda de la década de los años 70 y 80 se aceptaba la hipótesis de que cuanto más fuertes son las relaciones personales menor será el uso de los medios (obtrusive contengency), de modo que la experiencia personal sería un sustituto de los mensajes de los medios. Por último, cabe destacar la integración de la tradición teórica de la influencia personal en los estudios de agenda. Brosius y Weimann, tras citar los trabajos anteriores que habían relacionado las dos perspectivas, analizan el papel de los líderes de opinión en el proceso de agenda setting (1996: 566). Sus conclusiones vuelven a incidir en la complejidad de la explicación del proceso, al depender tanto del tipo de tema, el contexto y las características del público y en la capacidad de algunos individuos para influir en el efecto agenda en sus dos direcciones, de los medios al público y viceversa (1996: 675-77).

El tipo de medio ha sido uno de los factores más empleados a la hora de calibrar el efecto agenda (McCombs, 2004: 67; Zhu y Blood, 1996: 119). Por un lado, parece arriesgado generalizar la influencia de los medios tomando únicamente la prensa, porque si bien puede ser cierto que sea referente para los medios audiovisuales (agenda intermedios), no lo es menos que el consumo de información se concentra fundamentalmente en la televisión. A esta premisa de carácter cuantitativo, se debe añadir que diferentes medios pudieran producir efectos dispares, dado que los formatos audiovisuales requieren un procesamiento de la información distinto de los impresos (Shah, McLeod y Yoon, 2001; Wanta, 1997: 62). El tipo de medio ha de relacionarse necesariamente con sus usos, entendido en sus diversas vertientes de consumo o exposición, atención y aceptación; incluso con la credibilidad del medio como fuente de información. El consumo de medios ha 
sido empleado, además de en los estudios de agenda, como variable independiente o explicativa del conocimiento y/o comportamiento políticos. Por ejemplo, Norris (2000) rebate la idea de que la mayor exposición a los medios comporta pasividad frente a la vida política, señalando muy al contrario que el compromiso político está asociado a ciudadanos más informados. En nuestro estudio nos limitamos a incluir el nivel declarado de exposición a la información política y electoral durante el periodo de campaña a través de la prensa, la radio y la televisión. Más concretamente nos cuestionamos si cada tema será más o menos sensible al efecto agenda setting según el tipo de medio (hipótesis 3). No obstante, a pesar de que sin la exposición a los medios el efecto agenda es imposible, debe ser considerada en unión con otros factores psico-sociológicos (Wanta, 1997: 18-19).

Por último haremos algunas precisiones respecto a los temas de agenda, que nos parecen oportunas para nuestro trabajo. Conviene delimitar primero cuál de las distintas agendas será objeto de estudio. Aunque parezca obvio no siempre encontramos una definición clara y ello puede afectar al diseño de la investigación y a las conclusiones extraídas. McLeod, Becker y Byrnes (1974) categorizan la agenda pública en tres bloques: como agenda intrapersonal (cuál es el problema más importante para usted), como agenda interpersonal (cuál es el problema más importante para los demás individuos), y como agenda comunitaria (el problema más importante para el país). En nuestro caso, por el propio diseño del cuestionario trabajamos sobre dos aspectos de la agenda pública: los temas de preocupación personal y los temas de campaña. Para los primeros la pregunta fue: «Ahora voy a leerle una lista de temas que preocupan hoy a la gente. Me gustaría que me dijese ¿cuál es el que le preocupa a Ud. en primer lugar?». Los temas de campaña se extrajeron de las respuestas a la pregunta: «El pasado domingo 9 de marzo se celebraron elecciones generales. ¿Cuál cree que ha sido el tema más debatido por los partidos y candidatos a lo largo de la campaña electoral?» Los listados de asuntos que resultan de las respuestas son de naturaleza distinta, por cuanto la agenda personal englobaría aquellas preocupaciones que afectan directamente a la vida del individuo, mientras que la agenda de campaña se forma a partir de la percepción sobre los temas que han ocupado más a los actores políticos durante el periodo electoral. Este hecho provoca que las comparaciones entre ambas no sean sencillas, no sólo por lo que hemos señalado, sino porque en su construcción intervienen factores también dispares. Suponemos que entre ellos, los medios de comunicación jugarán un papel distinto.

La segunda cuestión relacionada con las agendas, ha llevado a considerar que los ciudadanos centran su atención en unos pocos temas, de modo que cada individuo pertenece a uno o más asuntos, estando relativamente bien informado sobre el que genera su interés y sobre el que realiza un seguimiento más o menos profundo en cuanto a las acciones de los políticos y de los partidos (Dalton, 1996: 111). Esta perspectiva requiere el análisis con datos individuales y no agregados, como en los estudios clásicos de agenda setting, por lo que hemos convertido cada tema en una única variable tipo dummy. 


\section{Diseño de la investigación}

El objetivo principal de este trabajo es abordar la hipótesis central del efecto agenda setting, es decir, qué papel juega el consumo de medios en la transferencia de la importancia otorgada a los temas de campaña, y además considerar las condiciones que pueden afectar al efecto agenda setting (Wanta, 1997: 3). Nuestra unidad de análisis es el individuo por lo que no utilizaremos datos agregados. Como ha señalado Wanta, «es el individuo quien consume y procesa la información contenida en los medios; es el individuo quien finalmente despliega el efecto agenda setting» (1997: 5). Al entender que además del consumo mediático, otros factores pueden explicar la transferencia de importancia, hemos optado por un modelo basado en la técnica multivariable de la regresión logística (método por pasos) que nos permita explicar cómo se comportan las distintas variables que acompañan a los medios de comunicación. En este sentido se ha utilizado, la encuesta postelectoral (2757) del Centro de Investigaciones Sociológicas (CIS), que se realizó entre el 12 de marzo y el 12 de mayo de 2008 con 6.083 entrevistas.

Obviamente, partimos del hecho de que a nivel agregado existe la evidencia del efecto agenda setting en las elecciones de 2008 en España. Puesto que el efecto está basado en el contenido de los medios (los temas que reciben mayor cobertura son percibidos como más importantes por los individuos), sólo si se detecta esta relación tiene sentido avanzar en otras explicaciones. Las correlaciones entre los temas de campaña más destacados por los medios y por los ciudadanos muestran niveles suficientes para confirmar la premisa. Los trabajos que se han venido publicando, procedentes del proyecto de investigación Voto racional y agenda mediática. Propuesta de seguimiento de la legislatura a través de grupos experimentales (Castromil, 2008; González y Bouza, 2009; Rodríguez y Castromil, 2009), muestran que los tres principales temas destacados en la prensa (economía, terrorismo e inmigración) ocupan también los tres primeros puestos en la agenda de campaña (tabla 3 ). «Este recuerdo de la campaña concuerda bastante bien con la agenda temática de los medios, por cuanto el terrorismo ocupaba el primer lugar entre los temas de la precampaña, tal como se desprendía de un estudio de las portadas y editoriales publicadas durante los tres meses previos a las elecciones» (González y Bouza, 2009: 189). Resultados muy similares se pueden deducir si se comparan los resultados del informe sobre la cobertura mediática de las elecciones realizado por profesores de la Universidad de Navarra (Pujol, 2008: 21) y los temas de campaña más citados.

A partir de la literatura existente abordamos las siguientes hipótesis:

H1: La importancia dada los temas de campaña se relaciona con las preocupaciones personales.

H2: Existe efecto agenda setting sobre los temas de campaña.

H3: Cada tipo de medio contribuye al efecto agenda en diferente proporción. 
H4: Existen temas de campaña más sensibles al efecto agenda.

Y una pregunta de investigación:

PI1: ¿Cómo interactúan las variables no relacionadas con los medios en el efecto agenda setting sobre los temas de campaña?

Las variables dependientes del estudio son los temas de campaña, resultantes de la pregunta: «El pasado domingo 9 de marzo se celebraron elecciones generales. ¿Cuál cree que ha sido el tema más debatido por los partidos y candidatos a lo largo de la campaña electoral?». Cada entrevistado tenía que nombrar un único tema, con respuesta espontánea, obteniéndose un listado de 29 temas. Para efectuar los análisis de regresión logística se procedió a recodificar sólo los seis primeros (terrorismo, ETA, problemas de índole económica, inmigración, vivienda, descalificaciones y paro) como una variable tipo dummy, aunque finalmente sólo se presentan los modelos para los tres primeros.

\section{Cuadro 1. Indicadores seleccionados para la regresión logística}

\begin{tabular}{|c|c|}
\hline & Categorías \\
\hline Primer tema que preocupa & 9 variables tipo dummy $(0-1)$ \\
\hline Sexo & $\begin{array}{l}\text { Hombre (1) } \\
\text { Mujer (0) }\end{array}$ \\
\hline Ideología & Escala izquierda-derecha (1-10) \\
\hline Estudios & Variable ordinal: primarios, secundarios y superiores (1-3) \\
\hline Recuerdo de voto & 5 variables dummy $(0-1)$ \\
\hline Interés en la campaña & Escala: ninguno-mucho (1-4) \\
\hline Opinión situación económica & Escala: muy mala-muy buena $(1-5)$ \\
\hline Opinión situación política & Escala: muy mala-muy buena $(1-5)$ \\
\hline $\begin{array}{l}\text { Relaciones personales y } \\
\text { discusión sobre la campaña } \\
\text { electoral }\end{array}$ & Escala: habitualmente-nunca o casi nunca (1-4) \\
\hline $\begin{array}{l}\text { Consumo información política } \\
\text { y electoral en TV }\end{array}$ & Escala: todos o casi todos los días-nunca o casi nunca (1-6) \\
\hline $\begin{array}{l}\text { Consumo información política } \\
\text { y electoral en prensa }\end{array}$ & Escala: todos o casi todos los días-nunca o casi nunca (1-6) \\
\hline $\begin{array}{l}\text { Consumo información política } \\
\text { y electoral en radio }\end{array}$ & Escala: todos o casi todos los días-nunca o casi nunca (1-6) \\
\hline $\begin{array}{l}\text { Consumo de propaganda } \\
\text { electoral }\end{array}$ & $\begin{array}{l}\text { Variable ordinal: } 0 \text { (ninguno), } 1 \text { (por televisión o por otro } \\
\text { material de campaña), } 2 \text { (por televisión y por otro material de } \\
\text { campaña) }\end{array}$ \\
\hline Comportamiento electoral & $\begin{array}{l}\text { Votó }(1) \\
\text { No votó }(0)\end{array}$ \\
\hline
\end{tabular}


La selección de las variables independientes atiende a la necesidad de ponderar la importancia relativa de la exposición a los medios respecto a otros aspectos. Para ello hemos considerado indicadores de tipo político (comportamiento electoral, ideología, recuerdo de voto, opinión sobre la situación política y económica); socio-demográficos (sexo y estudios); relacionados con la situación personal (relaciones personales y conversaciones sobre la campaña electoral y primer tema de preocupación personal); y específicos de la campaña electoral (consumo de propaganda electoral e interés en la campaña). Los indicadores sobre relaciones personales y discusión sobre la campaña electoral y el consumo de propaganda son dos variables formadas a partir de cuatro y dos variables respectivamente. El primer indicador es el resultado de la media de las cuatro variables originales, mientras que el segundo (ver cuadro 1) se construyó sumando los valores de las variables dicotómicas (sí-no): «Vio por televisión algún espacio de propaganda electoral de algún partido o coalición política» y «Leyó o echó un vistazo a cartas, folletos o programas de algún partido o coalición política».

\section{Resultados}

\subsection{La agenda personal y política, temas de campaña y cobertura mediática}

Durante los meses previos a las elecciones de marzo de 2008 los temas de la agenda se mantuvieron estables y ajustados a la clasificación de Tamayo y Carrillo (2005: 665-666) sobre la agenda pública de los españoles en crónicos (paro, terrorismo, problemas económicos), intermitentes (vivienda, problemas políticos) y nuevos (inmigración, violencia de género). La apropiación de los temas en función del partido político y la ideología, no revelan diferencias notables salvo en el caso de los problemas de índole económica, siempre más citados por los votantes del partido popular.

Tabla 1. Principal problema que existe actualmente en España (\%)

\begin{tabular}{|l|c|c|c|c|}
\hline & Enero & Febrero & Marzo & Postelectoral \\
\hline El paro & 20.1 & 21.8 & 21.4 & 28 \\
\hline Los problemas de índole económica & 17.1 & 17.2 & 19.5 & 17.6 \\
\hline El terrorismo, ETA & 16.3 & 13.6 & 15.4 & 23.4 \\
\hline La vivienda & 12.2 & 9.8 & 10.0 & 9.6 \\
\hline La inmigración & 8.4 & 10.4 & 8.4 & 3.4 \\
\hline La inseguridad ciudadana & 3.8 & 5.3 & 4.2 & 6.3 \\
\hline Las drogas & 0.9 & 0.9 & 0.7 & - \\
\hline $\begin{array}{l}\text { Los problemas relacionados con la calidad } \\
\text { del empleo }\end{array}$ & 3.6 & 3.0 & 2.3 & - \\
\hline La clase política, los partidos políticos & 4.4 & 3.8 & 3.5 & - \\
\hline
\end{tabular}

Fuente: CIS, barómetros de enero (2749), febrero (2754), marzo (2758) y postelectoral (2757) 
En la encuesta postelectoral, ante la pregunta sobre el problema que más preocupa, las respuestas vuelven a mostrar una ordenación muy similar con incrementos y descensos explicables bien por el efecto del desarrollo de algunos acontecimientos (por ejemplo, los atentados anteriores a las elecciones o el aumento del desempleo), o bien por la propia redacción de la pregunta en el cuestionario, que no se ajusta exactamente a la realizada en los barómetros. En esta ocasión hemos asociado los temas de preocupación más destacados con el indicador recuerdo y simpatía hacia un partido político. Ahora sí existen diferencias significativas en los casos del paro $(\chi 2=37,696 \mathrm{p} \leq 020)$, terrorismo $(\chi 2=$ $62,731 \mathrm{p} \leq 000)$, economía $(\chi 2=41,358 \mathrm{p} \leq 007)$, vivienda $(\chi 2=74,486 p \leq 000)$, sanidad $(\chi 2=38,855 \mathrm{p} \leq 015)$, educación $(\chi 2=60,403 \mathrm{p} \leq 000)$ y medioambiente $(\chi 2=59,463 \mathrm{p} \leq 000)$, es decir, los votantes y simpatizantes de ciertos partidos priorizan temas diversos. Los residuos tipificados corregidos revelan que a los votantes y simpatizantes del PSOE les preocupa el paro $(+2)$ y el terrorismo $(+3,9)$, a los del PP la economía $(+4,9)$ y a los de IU el medioambiente $(+4,3)$, la educación $(+3,7)$, la sanidad $(+2,4)$ y la vivienda $(+2,7)$.

Tabla 2. Temas de campaña (\%)

\begin{tabular}{|l|c|}
\hline El terrorismo, ETA & 28,0 \\
\hline Los problemas de índole económica & 21,8 \\
\hline La inmigración & 7,2 \\
\hline Todos los temas & 6,8 \\
\hline Ningún tema & 5,0 \\
\hline La vivienda & 1,5 \\
\hline El paro & 1,4 \\
\hline Las descalificaciones, insultos, etc. & 1,4 \\
\hline Otros temas &, 8 \\
\hline Las pensiones &, 7 \\
\hline La clase política, los partidos políticos &, 5 \\
\hline El Estatuto de Cataluña &, 5 \\
\hline Los problemas de índole social &, 3 \\
\hline Los problemas del agua, trasvase &, 3 \\
\hline Las guerras en general &, 2 \\
\hline La educación &, 2 \\
\hline La inseguridad ciudadana &, 1 \\
\hline Las infraestructuras &, 1 \\
\hline La violencia contra la mujer &, 1 \\
\hline Los problemas relacionados con la juventud &, 1 \\
\hline
\end{tabular}




\begin{tabular}{|l|c|}
\hline El Gobierno, los políticos y los partidos &, 1 \\
\hline Los nacionalismos &, 1 \\
\hline Los problemas relacionados con la mujer &, 1 \\
\hline N.S. & 21,3 \\
\hline N.C. & 1,4 \\
\hline Total & 100 \\
\hline
\end{tabular}

Fuente: CIS, postelectoral (2757)

La percepción de los temas de campaña propuestos y debatidos por los actores políticos presenta diferencias significativas respecto a las agendas pública y personal anteriores. Las preocupaciones ciudadanas apenas se verían reflejadas en la campaña electoral según los encuestados, con la excepción del terrorismo y la economía. En el grafico 1 -en el que se comparan las respuestas de los encuestados con la presencia de los temas también en la cobertura de los medios y en los comunicados de prensa de los dos partidos mayoritarios- observamos que los tres asuntos más destacados por los ciudadanos y en la cobertura mediática se acercan en la importancia otorgada, mientras que los temas de campaña no se ajustan a esta pauta. Si cruzamos los temas de campaña más destacados (tabla 2) con el indicador recuerdo y simpatía hacia un partido político encontramos diferencias significativas en los casos del terrorismo $(\chi 2=81,775$ $\mathrm{p} \leq 000)$, los problemas de índole económica $(\chi 2=83,318 \mathrm{p} \leq 000)$ e inmigración $(\chi 2=45,954 \mathrm{p} \leq 002)$, es decir, los votantes y simpatizantes de ciertos partidos priorizan temas diversos. Los residuos tipificados corregidos revelan que a los votantes y simpatizantes del PSOE les preocupa el terrorismo $(+6,2)$ y a los del PP los problemas de índole económica $(+5)$.

\section{Grafico 1. Agenda pública, de campaña y mediática (\%)}

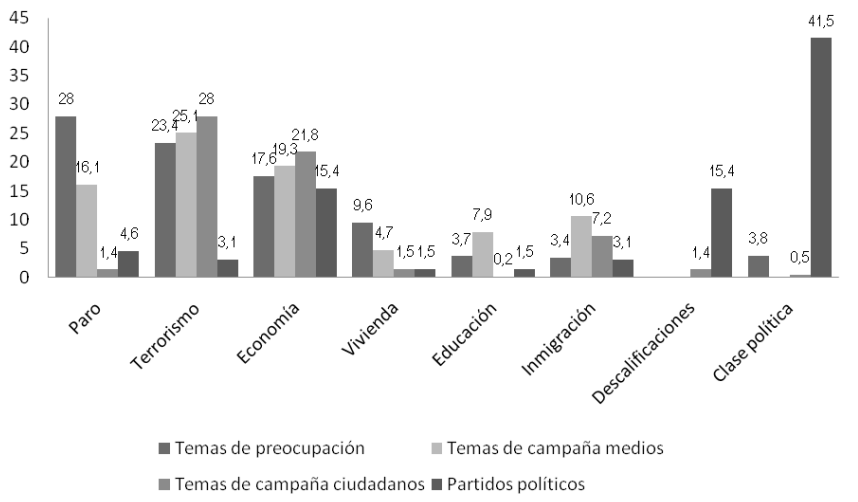




\subsection{Consumo de información y campaña electoral}

El conocimiento sobre la campaña electoral a través de los medios proviene fundamentalmente de la televisión, tanto en la información como en la propaganda electoral. Mientras que el 53,5\% de los encuestados declaraba haber seguido la campaña a través de la televisión todos o casi todos los días, los periódicos y emisoras de radio muestran la tendencia contraria (el $48,2 \%$ y el $61 \%$ respectivamente de las respuestas se concentran en la opción nunca o casi nunca). Lo mismo puede afirmarse del consumo de los mensajes electorales, donde predomina el canal televisivo $(63,8 \%)$ frente a los clásicos folletos, cartas o programas $(43,8 \%)$. Más concretamente, los informativos de TVE y los de Antena 3 suman el $57 \%$ del consumo.

El consumo de información sobre el proceso electoral estará probablemente relacionada con el interés por la propia campaña, que en general alcanza una media baja $(2,4$ sobre 4$)$. Así, tanto las tablas de contingencia como los residuos tipificados corroboran para televisión $(+15,4)$, radio $(+14,5)$ y/o prensa $(+15,5)$ que quienes manifiestan mucho interés en la campaña se informan con mayor regularidad. La hipótesis de Norris (2000) sobre la asociación entre interés por la política y exposición a la información quedaría confirmada con nuestros datos.

Junto a los mensajes de los medios y de los partidos políticos hemos considerado la función de la comunicación interpersonal. Los familiares y amigos son los interlocutores más habituales $(58,5 \%$ y $49,1 \%)$, mientras que los compañeros de trabajo y los vecinos lo son en mucha menor medida (27\% y $12 \%$, respectivamente). El interés por la campaña también se asocia positivamente con el volumen de conversaciones. En la regresión lineal construimos un índice conjunto $(\alpha$ de Cronbach $=, 720)$.

\subsection{Los factores explicativos de la atribución de importancia}

Las hipótesis que nos planteábamos en el estudio se han abordado con la técnica de la regresión logística, apropiada para las variables dependientes nominales. Con ella podemos analizar no sólo la relación entre cada tema de campaña y las variables predictoras seleccionadas, sino también el peso que cada una de ellas tiene en el modelo resultante. La regresión logística admite varios métodos de inclusión y exclusión de las variables, de los cuales hemos optado por el procedimiento por pasos, basado en la selección por criterios de significatividad estadística. De ahí que en los modelos resultantes sólo reflejemos las variables que contribuyen significativamente a explicar la varianza de la variable dependiente. Antes de comentar los resultados obtenidos, hemos de señalar que para ajustarnos a los supuestos de la regresión logística los temas vivienda, descalificaciones y paro no se han incluido al no contar con el número de casos mínimo (15 individuos por cada variable predictora) para ejecutar el procedimiento de 
máxima verosilimitud (Cea d'Ancona, 2004: 130). La tabla 3 muestra los modelos resultantes para los temas de campaña.

Antes de abordar las cinco hipótesis del trabajo, pasaremos a describir las variables explicativas de cada tema de campaña. La importancia del terrorismo y ETA vendría asociada con la preocupación por esta cuestión, el interés en la campaña y el consumo de televisión y la lectura de prensa, a los que se une la satisfacción con la situación económica. Que la ideología presente un signo negativo denota que quienes se sitúan más a la izquierda tienden a destacar el terrorismo como el tema principal del periodo electoral. La relevancia de los problemas económicos se explica fundamentalmente por la inquietud generada por la economía, la información televisiva y en prensa, las conversaciones interpersonales, la ideología - en este caso quienes se sitúan a la derecha- y el alto nivel de estudios. Por último, la mención de la inmigración se asocia con el sexo femenino, la preocupación por la inmigración, el voto al PSOE, la comunicación interpersonal y el alto nivel de estudios. Tenemos que destacar la relación negativa con la economía como tema, es decir, quienes mencionan la inmigración como el contenido más importante del discurso electoral no expresan preocupación por la economía.

La hipótesis primera establecía la posible relación entre temas de campaña y temas de preocupación. Suponíamos que los problemas más destacados de la agenda pública podrían relacionarse con la transferencia de importancia hacia los temas de campaña, aun cuando las frecuencias nos indicaban diferencias (Gráfico1). Efectivamente los coeficientes positivos siempre tienen mayor peso que los de las otras variables predictoras y, además, cada tema de campaña sólo se liga con su homónimo.

Las hipótesis 2 y 3 se centran en el efecto agenda setting sobre los temas de campaña y, en caso afirmativo, cómo contribuye cada tipo de medio al efecto agenda. Sólo para el terrorismo y los problemas de índole económica el consumo de información sobre la campaña en televisión y prensa es estadísticamente significativo, siendo mayor el peso de la televisión, que como ya hemos visto fue la fuente principal de conocimiento sobre la campaña. La inmigración se consideraría según nuestros datos dentro de los denominados obstrusive issues (hipótesis 4), es decir, aquellos más determinados por la experiencia directa. Como han señalado González y Bouza la inmigración es un tipo de problema relacionado con la experiencia personal (González y Bouza, 2009: 179). Pudiera sorprender que los problemas económicos no estén más relacionados con la opinión sobre la situación económica y el paro, teniendo en cuenta que el paro pasaba por considerarse una de las preocupaciones personales y el más importante problema del país. Una explicación plausible podría radicar en la reducción de este tema a las cuestiones macroeconómicas del momento (crisis del sistema financiero, etc.), lo que convertiría a la economía en un tema de campaña del tipo unobstrusive, aquellos que se ven menos afectados por la influencia de los medios (McCombs, 2004: 61-62). 
Para terminar, con el análisis multivariable pretendíamos calibrar el peso de los tres indicadores del consumo mediático, comparándolo con el resto de variables (PI1). En ninguno de los tres casos la información sobre el periodo electoral es el predictor más fuerte, e incluso para el tema inmigración no presenta un coeficiente estadísticamente significativo. Los modelos para los dos primeros temas de la tabla 3 apuntan a que el interés por la campaña se une la necesidad de más noticias sobre la misma, y en menor medida el posicionamiento ideológico (aún con sentidos contrarios). Un nivel de estudios alto explicaría la relevancia de los problemas de índole económica y la inmigración, así como las conversaciones interpersonales. Hay que destacar la escasa capacidad predictiva de las variables socioeconómicas y políticas, y la nula presencia de la propaganda electoral como detonante de la atribución de importancia.

Tabla 3. Modelos de regresión logística para los temas de campaña $(\operatorname{Exp}(B))$

\begin{tabular}{|c|c|c|c|}
\hline & Terrorismo, ETA & Problemas de índole económica & Inmigración \\
\hline Tema que más preocupa & $\begin{array}{l}1,434^{* * *} \\
\text { Terrorismo }\end{array}$ & $\begin{array}{l}1,783^{* 3 *} \\
\text { Economia }\end{array}$ & $\begin{array}{l}-, 700^{* *} \\
\text { Economía } \\
3,254^{* * *} \\
\text { Inmigración }\end{array}$ \\
\hline Sexo & - & - &,$- 716^{* * *}$ \\
\hline Ideología &,$- 938 * 8 *$ & $1,083^{* * *}$ & - \\
\hline Estudios & - & $1,376^{* * *}$ & $1,294^{* * *}$ \\
\hline Recuerdo de voto & - & - & $\begin{array}{l}1,264^{* *} \\
\text { PSOE }\end{array}$ \\
\hline Interés en la campaña & $1,117^{* *}$ & $1,262^{* * *}$ & - \\
\hline $\begin{array}{l}\text { Opinión situación } \\
\text { económica }\end{array}$ & $1,147^{* * *}$ & - & - \\
\hline $\begin{array}{l}\text { Relaciones personales y campaña } \\
\text { electoral }\end{array}$ & - & $1,189^{* * *}$ & $1,191^{* *}$ \\
\hline Consumo de información en TV & $1,088^{* * *}$ & $1,072^{* * *}$ & - \\
\hline Lectura de información en prensa & $1,053^{* * *}$ & $1,043^{* *}$ & - \\
\hline $\mathrm{N}$ & 1701 & 1326 & 436 \\
\hline $\begin{array}{l}\text { Pseudo R2/ } \\
\text { Prueba de Hosmer y Lemeshow }\end{array}$ & $\begin{array}{l}0,08 / \\
p \leq .304\end{array}$ & $\begin{array}{l}0,16 / \\
p \leq .752\end{array}$ & $\begin{array}{l}0,13 / \\
p \leq .174\end{array}$ \\
\hline$\%$ casos correctos & 69,4 & 75 & 92 \\
\hline
\end{tabular}

Elaboración propia. Se muestran los coeficientes estandarizados con niveles de significación $\leq .001^{* * *}, \leq .01^{* *}, \leq .05^{*}$.

\section{Conclusiones}

A pesar de las limitaciones que se derivan de haber trabajado con datos no recogidos ad hoc, la principal virtud del estudio es haber sometido a técnicas multivariables el efectos agenda setting para testar el peso de los medios y su interrelación con otros factores en el nivel micro. Generalmente los análisis bivariados con muestras grandes como la nuestra tienden a ofrecer relaciones significativas, pero sólo cuando planteamos modelos multivariables conseguimos calibrar mejor dichas relaciones. Como señalábamos en el marco teórico nuestro objetivo no se centraba en las relaciones de causalidad, sino en la búsqueda de variables que nos ayuden a comprenden la imputación de importancia sobre los temas de la campaña electoral. Encontrar un modelo no es una tarea fácil en las ciencias sociales, más en nuestro caso en el que la bibliografía existente no 
es concluyente. Por ello, y a pesar de que la aplicación de la técnica de análisis elegida es más potente cuando las variables predictoras no son numerosas, optamos por incluir en el diseño hasta catorce factores (cuadro 1) y que el método por pasos de la regresión logística actuara de selector estadístico.

Así, hemos testado tanto la perspectiva de la llamada contingent theory, o de los determinantes del efecto agenda, como la que sostiene la importancia del contexto. Nuestros resultados apoyan que el papel del consumo de información ha de conjugarse con otras variables, entre las cuales destacamos la agenda personal por encina de cualquiera de las otras características, dado que este indicador es el único que comparten los tres temas. Por otra parte, nos ha sorprendido que la influencia de los distintos formatos de propaganda electoral sobre la mención de los tres temas de campaña es muy escasa, lo cual confirmaría la necesidad de los partidos y líderes por vehicular sus mensajes electorales a través de los medios tradicionales para garantizarse una mayor eficacia (Humanes, 2009: 109).

Debemos resaltar que hemos corroborado la mayor sensibilidad a la influencia del consumo de medios de los temas menos apegados a la experiencia. En este sentido, nuestro estudio vendría a sumarse a lo concluido por la literatura existente en diversos contextos, y también en el caso español (Canel, Llamas y Rey, 1996). Como señalábamos en el primer apartado, en los obstrusive issues (inmigración), la comunicación interpersonal cobra mayor relevancia que los medios. Los problemas de índole económica presentan una situación en la que las interacciones personales y el consumo de información forman parte del modelo predictivo. Al no haber diseñado un análisis de causalidad no podemos determinar cuál de los dos factores motiva el otro, pero nos abre una vía de estudio interesante. Por último, para el terrorismo la televisión y la prensa son los únicos canales de conocimiento.

\section{Referencias}

Brians, Craig y Wattenberg, Martin (1996). Campaign issue knowledge and salience: Comparing reception from TV commercials TV news, and newspapers. En: American Journal of Political Science 43, 1189-1208.

Brosius, Hans Bern y Weimann, Gabriel (1996). Who sets the agenda?: Agenda setting as a two step flow. En: Communication Research 23, 561-580.

Castromil, Antón R. (2008). El negativismo mediático en las Elecciones Generales de 2008 en España. En: Estudio/Working Paper 2008/1. Revista On Line del G.T Walter Lippmann.

[http://www.ucm.es/info/socvi/gtsocvi/paginas/working_papers.htm].

Cea d’Ancona, M. Ángeles (2004). Análisis multivariable. Teoría y práctica en la investigación social. Madrid: Síntesis. 
Russell, Dalton (1996). Citizens Politics. Public Opinion and Political Parties in Advanced Western Democracies. Chatham: Chatham House.

Demers, David (1993). Community attachment, social priming and newspaper. Paper presented at the Annual Conference of the Association for Education in Journalism and Mass Communication (11-14 August).

Demers, David et al. (1989). Issue Obtrusiveness and the Agenda-Setting Effects of National Network News. En: Communication Research 16, 793-812.

Dunaway, Johanna; Abrahano, Marisa y Branton, (2007). Agenda Setting, Public Opinion, and the Issue of Immigration Reform. Working Paper 162, February. San Diego: The Center for Comparative Immigration Studies, University of California.

González, Juan Jesús y Bouza, Fermín (2009). Las razones del voto en la España democrática, 1977-2008. Madrid: Los libros de La Catarata.

Golan, y Goidel, Robert Kirby (2004). Attribute Agenda Setting and the Media: Measuring exposure as a contingent condition Public Issue Priority Formation: Media Agenda-Setting and Social Interaction. Paper presented at the annual meeting of the International Communication Association, New Orleans Sheraton, New Orleans, LA Online.[http://www.allacademic.com/meta/p112989_index.html].

Humanes, María Luisa (2009). Nuevos temas noticiosos en las elecciones generales de 2004. La presencia de metacobertura en la prensa española. En: Zer $26,105-128$.

Iyengar, Shanto y Kinder, Donald (1987). News that matter. Chicago: University of Chicago Press.

Kosicki, Gerald (1993). Problems and opportunities in agenda-setting research. En: Journal of communication 43, 100-127.

McCombs, Maxwell E. (2004). Setting the agenda: The mass media and public opinion. Cambridge: Polity Press.

McLeod, Jack; Becker, Lee y Byrnes, James (1974). Another look at the agendasetting function of the press. En: Communication Research 1, 131-166.

Norris, Pipp (2000). A virtuous circle: Political communications in postindustrial societies. Cambridge: Cambridge University Press.

Protess David L. et al. (1985). Uncovering rape: the wachtdog press and the limits of agenda-setting. En: Public Opinion Quarterly 49, 19-37.

Pujol, Francesc (2008). Informe sobre la cobertura mediática de las elecciones al Parlamento español del 9 de marzo de 2008. Informe R-01. Pamplona: Politics and Media Value Project, Universidad de Navarra. 
[http://www.unav.es/.../1205067116_Informe_Cobertura_Elecciones_2008.pdf]. Rodríguez, Raquel y Castromil, Antón (2009). Encuadres periodísticos y campañas electorales: transmisión, influencia y atribución de responsabilidad. Ponencia presentada en el IX Congreso de AECPA: Repensar la democracia: inclusión y diversidad.

[http://www.aecpa.es/congresos/congreso_09/grupos-trabajo/area03/GT02/actas.php].

Shah, Dhavan; McLeod, Jack y Yoon, So-Hyang (2001). Communication, context and community: an exploration of print, Broadcast and internet influences. En: Communication Research 28, 464-506.

Soroka, Stuart et al. (2009). Campaign News and Vote Intentions. En: Journal of Elections, Public Opinion and Parties 19, 359-376.

Tamayo, Manuel y Carrillo, Ernesto (2005). La formación de la agenda pública. En: Foro Internacional XLV, 658-681.

Walgrave, Stefaan; Soroka, Stuart y Nuytemans, Michiel (2008). The Mass Media's Political Agenda-Setting Power A Longitudinal Analysis of Media, Parliament, and Government in Belgium (1993 to 2000). En: Comparative Political Studies, vol. 41, $\mathrm{n}^{\circ} 6,814-836$.

Walgrave, Stefaan y Van Aelst, Pe (2006). The Contingency of the Mass Media's Political Agenda Setting Power: Toward a Preliminary Theory. En: Journal of Communication 56, 88-109.

Walgrave, Stefaan y Van Aelst, Peter (2004). The mass media's political agendasetting power. Towards an integration of the available evidence. Prepared for delivery at the APSA political communication pre-conference, Chicago. [http:// www. tigger.uic.edu/depts/pols/PolComConf/Walgrave.pdf].

Wanta, Wayne (1997). The public and national agenda. How people learn about important issues. Mahwaa: Laurence Erlbaum.

Zhao, Xinshu y Chaffee, Steven (1995). Campaign advertisements versus television news as sources of political issue information. En: Public Opinion Quarterly 59, 41-65.

Zhu, Jian-Hua Jonathan y Blood, Deborah (1996). Media Agenda-Setting Theory: Review of a 25-Year Research Tradition. En: Hong Kong Journals on line. [http://sunzi.lib.hku.hk/hkjo/view/10/1000100.pdf].

Zhu, Jian-Hua Jonathan et al. (1993). Public Issue Priority Formation: Media Agenda-Setting and Social Interaction. En: Journal of Communication 43, 8-29. 


\section{Referencia de este artículo}

Humanes, María Luisa (2012). El efecto agenda sobre los temas de campaña en las elecciones generales de 2008. En: adComunica. Revista Científica de Estrategias, Tendencias e Innovación en Comunicación, $\mathrm{n}^{0}$ 3. Castellón: Asociación para el Desarrollo de la Comunicación adComunica, Universidad Complutense de Madrid y Universitat Jaume I, 191-207. DOI: http://dx.doi.org/10.6035/21740992.2012 .3 .12 